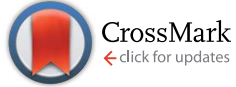

Cite this: Soft Matter, 2014, 10, 6237

\title{
Ordered liquids and hydrogels from alkenyl succinic ester terminated bola-amphiphiles for large-scale applications $\uparrow$
}

\begin{abstract}
Thomas Lohmeier, ${ }^{a}$ Michael Bredol, ${ }^{b}$ Eduard Schreiner ${ }^{c}$ and Horst Hintze-Bruening ${ }^{\star a}$
The present study describes an economic and scalable approach to aqueous mesophases from bolaamphiphiles (BA) obtained via nucleophilic addition of dimer fatty acid based $\alpha, \omega$-polyesterdiols (PES) on cyclic acid anhydrides and conversion of the carboxylic end groups into ammonium salts. Novel bolaamphiphilic head groups are introduced using alkenyl succinic anhydrides (ASA). The additional terminal hydrophobic side chains favour the self-assembly of polymeric BA of different molecular weights into nanoscale anisotropic objects, their shape and ordering into nematic or lamellar-like phases being dependent on the length and structural uniformity of the ASA chains. Corresponding diester based on C15 (hydrogenated bisphenol-A, HBA) and C8 (1,4-cyclohexanedimethanol, CHDM) spacers have been prepared and the self-assembly of the resulting BA in water has been studied using SAXS, ${ }^{2} \mathrm{H}-\mathrm{NMR}$ and optical polarization microscopy. While the rigid C8 spacer impedes any ordering, ASA capped C15 tends to form ordered hydrogels over extended regions of the phase diagram that resemble mesh phases and $L_{\alpha} / L_{3}$ polymorphism. Rheological and simulation results confirm the presence of elastically responding bicontinuous morphologies and biased porous assemblies resembling interconnected mesh phases. Both the use of the dimer fatty acid based spacer as well as of ASA head groups open up large-scale applications of ordered liquids (or hydrogels) as a formulation basis for e.g. films, coatings and adhesives.
\end{abstract}

Received 20th May 2014

Accepted 13th June 2014

DOI: $10.1039 / \mathrm{c} 4 \mathrm{sm} 01102 \mathrm{c}$

www.rsc.org/softmatter hydroxide adopt a nematic like ordering in the aqueous phase. ${ }^{5}$ However the limited extension of nematically arranged platelets perpendicular to the director led to a superimposed lamellar assembly on larger length scales which was transferred into the solid coating in the form of an ordered array of polymer intercalated platelets. In this study we describe an approach to obtain lyotropic ordering of polymeric bola-amphiphiles in the aqueous phase, namely extended layered structures, thus mimicking robust lipid membranes of archaebacteria that consist of bola-amphiphiles, a sort of amphiphiles comprising two hydrophilic heads connected by a hydrophobic spacer.

Since early studies on bola electrolytes ${ }^{6}$ and on cationic bolaform detergents ${ }^{7}$ as well as the pioneering investigations of Vittorio Luzzati on the polymorphism of several extracted archae-bacterial bola-amphiphiles ${ }^{8}$ numerous synthetic systems have been prepared and their self-assembly studied, ${ }^{9}$ the main aspects investigated being the nature of the head groups (non-, an- or cationic) and the spacer (aliphatic, aromatic, and fluorinated), the molecular architecture (symmetry, length, bulkiness, and shape) or the concept of supra-amphiphiles featuring specific binding sites and the role of the counter ion. ${ }^{10}$ Common to all is the molecular uniformity (purity) of the organic mesogen resulting from tedious synthesis and purification procedures that are economically not affordable for large-scale industrial application. Furthermore low molecular weights and thus high proportions of hydrophilic, 
possibly ionic groups are incompatible with coating applications which are thought to provide resistance against humidity with regard to swelling and (ion) permeability.

We found that ill-defined bola-form polyesters prepared from inexpensive raw materials self-assemble in aqueous solutions into ordered structures and could thus overcome the economic and technological constraints. Besides hydrophobicity stemming from dimer fatty acids in the polymer backbone we have investigated the effect of hydrophobic aliphatic side groups in the $\alpha$ - and $\beta$-position to the ionic head group on the self-assembly. The role of double-stranded bolaform architecture has already been addressed in the context of vesicle formation of symmetrical glycosylated bola-amphiphiles ${ }^{\mathbf{1 1}}$ making reference to similar asymmetric molecules where hydrophobic chains were attached at the anomeric site of the head groups' furanose ring. ${ }^{12}$ In the present study the head groups result from nucleophilic addition of an $\alpha, \omega$-polyesterdiol (PES) on alkenyl succinic anhydrides (ASA) which have been proposed for numerous technical applications and are used on a large scale, e.g. as sizing agents for $\operatorname{starch}^{\mathbf{1 3}}$ and cellulose $^{\mathbf{1 4}}$ in the food and paper industry respectively. Contrary to succinic and maleic anhydride head groups ${ }^{\mathbf{1 5}}$ as well as dimer fatty acids ${ }^{\mathbf{1 6}}$ - commercially available as technical mixtures of isomers on the ton scale - ASA derived head groups have not yet been proposed for the synthesis of bola-amphiphiles to the best of our knowledge.

This article is divided into four parts. The first chapter describes the synthesis and molecular diversity of the polymeric bola-amphiphiles. The formation and characterization of their aqueous dispersions are given in the second chapter. The third section addresses the role of different ASA derived terminal side chains, which was studied in monomeric diester based bolaamphiphiles. Finally the systems studied are put into the context of shorter homologues that resemble Gemini surfactants as well as non-bola type amphiphilic polyesters.

\section{Experimental}

\section{Materials}

Distilled dimer fatty acid A2 [CAS 61788-89-4, 3.5 meq acid, PRIPOL 1012, Croda Ltd.], hydrogenated bisphenol-A [CAS 8004-6, 7.92 meq hydroxyl, Aceto Corp., USA], 1,4-bis (hydroxymethyl)cyclohexane [CAS 105-08-8, 99\%, Sigma-Aldrich], hydrogenated phthalic anhydride A1 [CAS 85-42-7, 95\%, Sigma-Aldrich] and dimethyl ethanolamine [CAS 108-01-0, $>98 \%$, Sigma-Aldrich] were used as supplied. Succinic anhydride [CAS 108-30-5, 99\%, DSM], maleic anhydride [CAS 10831-6, 99\%, Sigma-Aldrich], trimellitic anhydride [CAS 552-30-7, $>97 \%$, Sigma-Aldrich], alkenyl succinic anhydride 2c [CAS 26680-54-6, >97\%, Trigon], 2d [CAS 19780-11-1, 95\% SigmaAldrich], 2e [CAS 25377-73-5, >96\%, Merck], 2f [CAS 26544-387 , $>98 \%$, Trigon] and $2 \mathrm{~g}$ [mixture of CAS 28777-98-2 and CAS 32072-96-1, $>93 \%$, Trigon] were used as obtained. Butan2-one [CAS 78-93-3, 99.5\%] was dried over a molecular sieve (4 A) for $24 \mathrm{~h}$ before use. Deionized water was used throughout all experiments.

\section{Synthesis}

Polyester 1 (PES) was synthesized from an equimolar mixture of $\mathbf{A 1}$ and $\mathbf{A} 2$ together with hydrogenated bisphenol-A using a diol to diacid ratio of 2.26 under nitrogen and stirring at $190{ }^{\circ} \mathrm{C}$ and distillation of water until an acid functionality below $0.15 \mathrm{meq}$ of the reaction mixture was reached. Polyester PES-NB was obtained from the addition-condensation reaction of $72.7 \mathrm{~g} 1$ (2.55 meq hydroxyl) with $8.35 \mathrm{~g}$ trimellitic anhydride under nitrogen and stirring and water distillation at $160{ }^{\circ} \mathrm{C}$ until an acid functionality of $0.62 \mathrm{meq}$ was reached. For the bolaamphiphiles 3, 4 and 5, the polyester 1, hydrogenated bisphenol-A and 1,4-bis (hydroxymethyl)cyclohexane respectively were reacted with sub-stoichiometric amounts of 0.9 equivalents cyclic anhydride 2 per hydroxyl at $95{ }^{\circ} \mathrm{C}$ in 2-butanone under stirring and under dried nitrogen until 100\% anhydride conversion according to titrations under aqueous versus ethanolic conditions.

\section{Dispersion}

All polymeric amphiphiles 3 and PES-NB were neutralized using 0.95 equivalent dimethyl ethanolamine per acid at $80^{\circ} \mathrm{C}$ followed by addition of water and distillation of 2-butanone until a content $<0.5$ weight $\%$ was reached. Water was added to adjust the amphiphile concentration, expressed as the $w$ value for the ammonium salts, before the mixture was cooled to ambient temperature. For amphiphiles 4 and 5 the same procedure was applied using a minimum temperature of $60^{\circ} \mathrm{C}$.

\section{Characterization}

Small-angle X-ray scattering (SAXS) was recorded on the ID02high brilliance beamline of ESRF, Grenoble, France using monochromatic radiation $(\lambda=0.1 \mathrm{~nm})$, a source-sample distance of $55 \mathrm{~m}$ and sample-detector distances (max range 1 to $10 \mathrm{~m})$ to cover a typical $q$-range of 0.3 to $4.3 \mathrm{~nm}^{-1}(0.03$ to $6 \mathrm{~nm}^{-1}$ for $\mathbf{3 a}$ and $\mathbf{3 b}$ ). Intensities were corrected for empty mica platelets that were used as sample holders.

${ }^{2} \mathrm{H}-\mathrm{NMR}$ spectra were recorded with an Agilent VNMRS 500 $\mathrm{MHz}$ spectrometer at a resonance frequency of $76717 \mathrm{MHz}$, with a spectral width of $7700 \mathrm{~Hz}, 80$ transients, a pulse width of $20 \mu \mathrm{s}(\pi / 2)$ and an acquisition time of $0.3 \mathrm{~s}$. Samples were preequilibrated for $10 \mathrm{~min}$. The heating rate was $2.5^{\circ} \mathrm{C} \mathrm{min}{ }^{-1}$.

Polarization light microscopy (PLM) pictures were taken by using an Olympus BX51 microscope equipped with an UM Plan FI-lens and a XC10 digital camera. A droplet of the sample, conditioned at room temperature was prepared between a glass slide and a cover slip. For temperature-dependent PLM a Leitz Orthoplan light microscope equipped with a Linkam temperature control unit and a liquid nitrogen cooling unit LNP, TMS 94 was used.

Cryogenic atomic force microscopy (cryo-AFM) was carried out using a Bruker Dimension Icon equipped with a FastScan ScanAsyst scanner and a Veeco DI TA controller. Samples were prepared using a Reichert Ultracut $S$ sectioning system equipped with a FCS cryo extension. A droplet of the sample, conditioned under ambient conditions for $10 \mathrm{~min}$, was deposited on 
a metal attachment and microtomed at $-80{ }^{\circ} \mathrm{C}$. The frozen, truncated sample was transferred to the precooled stage $\left(-20{ }^{\circ} \mathrm{C}\right)$. AFM recording started after complete sublimation of formed ice crystals (approx. $15 \mathrm{~min}$ ) using a frequency of $15 \mathrm{~Hz}$.

Cryogenic transmission electron microscopy (cryo-TEM) was performed on a FEI Tecnai $\mathrm{G}^{2}$ electron microscope using $200 \mathrm{kV}$ as the accelerating voltage. Ultrathin sections $(70 \mathrm{~nm})$ of the sample were cut at $-80{ }^{\circ} \mathrm{C}$ using a Leica UC6 ultra-microtome equipped with a Leica EM FCS cryo chamber and a diamond knife.

Rheological characterization of the bola-amphiphiles 4 was performed on an Anton Paar MCR302 CP25/ $1^{\circ}$ rheometer for amplitude and frequency sweeps at $23{ }^{\circ} \mathrm{C}$ using a cone plate distance of $0.05 \mathrm{~mm}$ at a constant frequency of $1 \mathrm{~Hz}$ and a constant amplitude of $0.1 \mathrm{mrad}$ covering $8.8 \times 10^{-3}$ to $1000 \mathrm{mrad}$ and 1 and $100 \mathrm{~Hz}$ respectively. Temperature dependent measurements were performed on an Anton Paar MCR501 CP50/1 ${ }^{\circ}$ rheometer using a cone plate distance of $0.099 \mathrm{~mm}$ at a constant frequency of $1 \mathrm{~Hz}$ and an amplitude of $5.8 \mathrm{mrad}$ and applying a constant heating rate of $1 \mathrm{~K} \mathrm{~min}^{-1}$ covering $20-90^{\circ} \mathrm{C}$.

Rheological characterization of PES-NB was performed using a Haake MARS system (Thermo Scientific) with a $2^{\circ}$ titanium cone and a glass plate (gap width $70 \mu \mathrm{m}$ ), and temperature-controlled by using a Haake thermostat. For temperature dependent measurements appropriate steps were defined. Only data that fulfill the harmonic approximation after stabilization $(\Delta T<1 \mathrm{~K})$ were used. These were taken at 10 $\mathrm{Pa}$ and $100 \mathrm{~Pa}$ and a frequency of $1 \mathrm{~Hz}$ over a period of at least 3 min on each stress level. Due to high viscosity of PES-NB dispersions the gap might not always be filled completely in its outer part. Therefore absolute values of $G^{\prime}$ and $G^{\prime \prime}$ may be offset and should be compared directly only in individual measurement runs.

Simulated systems contained various isomers of $\mathbf{4 c}$, counter ions and water yielding a total $N=16000$ atoms their proportions adjusted to concentrations of the organic phase (4c plus counter ions) in the range of $0.3 \leq w \leq 0.6$. All molecular dynamics simulations were carried out using LAMMPS $\ddagger$ (1) within an NPT ensemble, i.e. at constant pressure and temperature ( $p=1 \mathrm{~atm}, T=298.15 \mathrm{~K}$ ) and an integration time step of one femtosecond. Interatomic potentials were described by the COMPASS $\S$ force field and VMD 9 was used for analysis and visualization.

\section{Results and discussion}

\section{Amphiphilic polyesters: synthesis and structure}

All polymers are based on a hydrophobic $\alpha, \omega$-polyesterdiol (PES) which is obtained from the esterification between hydrogenated bisphenol-A (HBA) and a $1: 1$ molar mixture of

\$J. Comp. Phys., 1995, 117, 1; Sandia National Laboratories, Large-scale Atomic/Molecular Massively Parallel Simulator (LAMMPS 1995-2011), http://lammps.sandia.gov.

$\S$ J. Phys. Chem. B, 1998, 102, 7338.

ฯ J. Mol. Graphics, 1996, 14, 33. hydrogenated phthalic anhydride (HPA) and dimer fatty acid (DFA). Nucleophilic addition of the terminal hydroxyl groups on a cyclic anhydride (SA, MA, ASA, and TMA) converts PES into a telechelic carboxylic acid terminated polyester, namely a polymeric bola-amphiphile PES-BA (Fig. 1). For the non-bola polyester PES-NB ring opening of TMA is followed by polycondensation which formally yields chain extended PES with residual mid chain carboxylic acid groups. Molecular weight distributions from SEC (Fig. 2a) show that PES 1 consists of numerous different species reflecting both the mass and size difference between the C8 and C36 based synthons A1 and A2 respectively as well as a different reactivity between HPA (anhydride) and DFA (free acid) towards the hydroxyls of HBA. The latter was chosen because of its combined hydrophobicity, stiffness and hydrolytic stability of the formed ester bond. However this goes along with a low reactivity in the esterification and anhydride ring opening which entails prolonged synthesis compared to primary alcohol esterification. For compensation we used an unusually high diol to diacid ratio (2.26) that in theory does not allow polymer formation. Without further analytical evidence relating to the chemical nature of the different SEC fractions it is evident that PES must consist of oligomers besides diester and excessive HBA. This is corroborated by the size exclusion chromatogram of $\mathbf{4 c}$ which is shown for comparison in Fig. 2b. Thus non-equilibrium reflects kinetically favoured esterification between HPA (ring opening) and "trans" isomers of HBA (least steric hindrance). It has to be noted that the HBA quality we used consists of three isomers with respect to the hydroxyl ring position. Related to bisequatorial configured isopropylidene moieties equatorial and axial positioned hydroxyl groups account for a dominating trans,trans isomer $(w=0.5)$, a cis,trans isomer and a minor portion $(w=0.1)$ of a cis,cis isomer (see ESI $\dagger$ ). Assuming the fastest reaction of trans, trans HBA with HPA due to least steric hindrance and ring opening and a higher reactivity of DFA compared to that of the HPA-HBA addition product (steric hindrance in the latter), the most probable intermediate would be HPA-HBA-DFA which in turn would preferentially consume trans,trans-HBA followed by trans, cis-HBA. Thus residual HBA should consist of enriched cis,trans- and cis, cis-HBA. These most probable species are highlighted in a list of all combinatory conceivable bola-amphiphiles of exemplary $\mathbf{3 c}$ indicating molecular weights and lengths in all-trans configuration (Table $\mathrm{S} 1 \dagger)$. Molecular diversity in PES additionally stems from nonuniformity of DFA that comprises numerous isomers regarding the 'connecting' moieties, best described as products of an enereaction and a cycloaddition. ${ }^{\mathbf{1 6}}$ Thus linear and cyclic increments with varying proportions of saturated aliphatic, unsaturated and aromatic bonds depending on process parameters and optional post hydrogenation are common. The quality we used comprises minor amounts of mono- and tri-functional species ( $c f$. Experimental part). Regarding the SEC curves (Fig. 2) we emphasize that no absolute molecular weights can be deduced due to PMMA standards. However comparing distributions of PES with different PES-BA (3a, 3c) and diester (4c) it is obvious that the prominent peaks fall into the range of theoretically deduced molecular weights (vide supra, $c f$. 
Table S1 $\dagger$ ). Noteworthy the shape of the curves found for $3 \mathbf{a}$ and $3 \mathbf{c}$ indicates an impact of the head groups on the hydrodynamic radius. As expected for PES-NB, the mean molecular weight and the dispersity $\left(M_{\mathrm{w}} / M_{\mathrm{n}}\right)$ are considerably higher (Fig. 2c).

Regarding the amphiphiles of series 3 and 4 molecular complexity increases after ASA and TMA ring-opening which yields ester groups bearing an alkenyl chain in the $\alpha$ - or $\beta$ position or the pristine carboxylic acid group in the para or meta position respectively ( $c f$. Fig. 1). Finally for a given ASA type branching and cis/trans isomerism of the aliphatic group adds to molecular plurality.

Therefore we studied the impact of the length (C8, C12, C16) C18) and the structure (linear, branched) of the alkenyl side group. Numerous different ASA qualities are commercially available. Typically synthesized from maleic anhydride and $\alpha$ olefins in an ene-reaction it is known that mixtures of branched products are obtained under process conditions where the olefinic double bond is prone to migrate chain inwards. ${ }^{17}$ Thus at least three different materials are labelled "dodecenyl-SA": a rather pure chemical comprising two (cis,trans) linear C12 chains (2d), a mixture of isomers with respect to branched C12
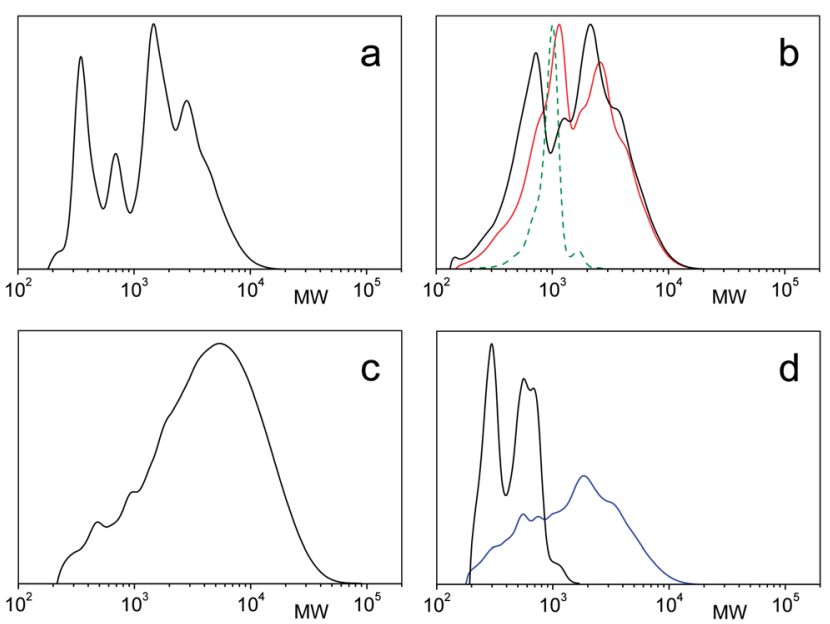

Fig. 2 Molecular weight distributions of (a) PES (1), (b) polymeric bolaamphiphiles $3 \mathrm{a}$ (black), 3c (red) and diester bola-amphiphile 4c (green, dashed), (c) non-bolaform PES-NB, and (d) separated lamellar phase (black) and isotropic phase (blue) of polymeric BA $3 a(w=0.54)$.

$$
\begin{aligned}
& 3 \text { a R1 = H } \\
& 3 \mathrm{c} \mathrm{R} 1=\mathrm{C} \text { linear } \\
& 3 \mathrm{e} \mathrm{R} 1=\mathrm{C} 12 \text { bran } \\
& 3 \mathrm{f} \mathrm{R} 1=\mathrm{C} 12 \text { tetra } \\
& 3 \mathrm{~g} \mathrm{R} 1=\mathrm{C} 16 / \mathrm{C} 18
\end{aligned}
$$

3 e R1 $=$ C12 branched

3 f R1 = C12 tetrapropenyl

3 g R1 $=$ C16/C18 (isomers)

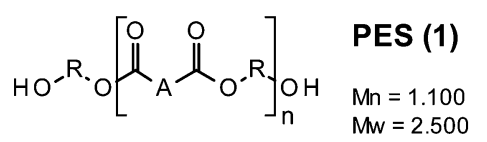

$\mathrm{R}=\mathrm{C} 15$ (hydr. Bisphenol-A)

$$
\begin{aligned}
& 4 \text { a R1 }=H \\
& \text { 4 c R1 }=\mathrm{C} 8 \text { linear } \\
& \text { 4 d R1 }=\mathrm{C} 12 \text { linear } \\
& \text { 4 e R1 }=\mathrm{C} 12 \text { branched }
\end{aligned}
$$

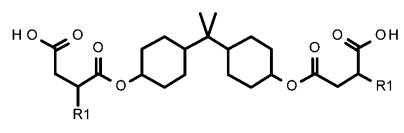

$4 \mathrm{~h}$ trimellithic

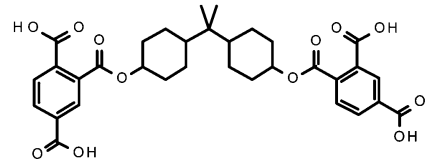

diester BA (4)

cyclic acid anhydrides $2(a-h)$

MEK, $80^{\circ} \mathrm{C}$, reflux<smiles>CC(C)(C1CCC(O)CC1)C1CCC(O)CC1</smiles>

hydrogenated Bisphenol-A dimer fatty acid, mixture of isomers (A1)

hydrogenated phthalic anhydride (A2)

bulk, $190^{\circ} \mathrm{C}$, minus $\mathrm{H}_{2} \mathrm{O}$
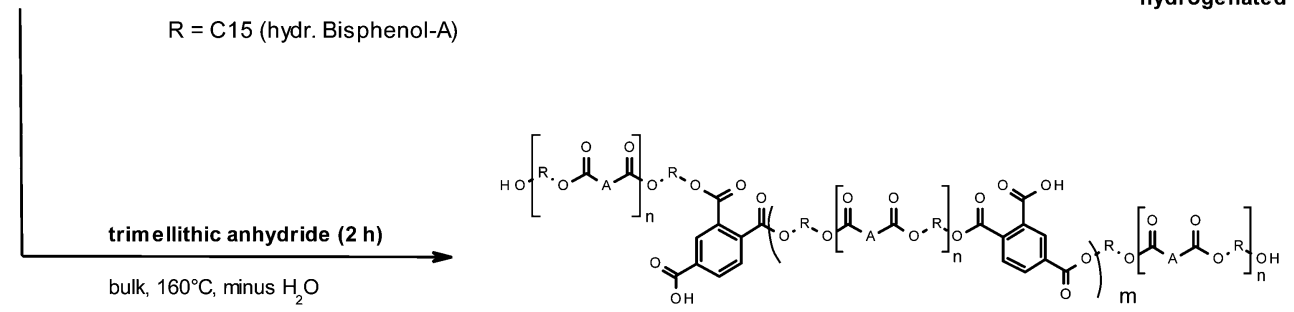

PES-NB

$\mathrm{Mn}=1.900$

$\mathrm{Mw}=5.900$

Fig. 1 Survey of synthesized polymeric bola-amphiphiles (3) based on the $\alpha, \omega$-polyesterdiol PES (1), hydrogenated bisphenol-A derived diester bola-amphiphiles (4) and the non-bola-amphiphile PES-NB obtained from (1) in an addition-polycondensation process. Increment ' $A$ ' in (1) symbolizes a mixture of A1 and A2 (see Experimental part). For structures ( 3 ) and (4) with $\mathrm{R} 1 \neq \mathrm{H}$ and trimellitic head group asymmetric molecules represent isomerism with $\mathrm{R} 1$ in the $\alpha$ - or $\beta$-position and the pristine $\mathrm{CO}_{2} \mathrm{H}$ group in the meta or para position respectively (see text). Replacement of HBA by the 1,4-bis(hydroxymethyl)cyclohexane (CHDM) corresponding diester has led to the synthesis of $2 \mathrm{a}, 2 \mathrm{c}$ and $2 \mathrm{e}$ respectively, that are omitted for clarity. 
chains stemming from an undefined precursor or an in situ scrambled linear $\alpha$-olefin (2e) and finally an isomer mixture based on tetrapropenyl (2f). While the short C8 ASA (2c) consists of two linear species (cis,trans), GC analysis of C16/C18 ASA (2g) shows two sets of numerous isomers for the two different chain lengths (not shown). We compared these ASA terminated PESBA with those bearing succinic (2a), maleic (2b) half ester as well as the addition product from TMA (2h).

\section{Mesophases from PES-BA}

For dispersion into water the amphiphile acidic groups were transformed into the ammonium salt of dimethyl ethanolamine. The PES-BA (3a-3h) are dispersed in water covering a concentration range of $0.3<w<0.8$ ( $w=$ weight fraction of PESBA). For this purpose samples were taken from a dilution process of a concentrated stock solution $(w \sim 0.8)$ at $80{ }^{\circ} \mathrm{C}$. However with succinic and maleic terminated PES-BA (3a, 3b) homogeneous mixtures could not be obtained for mixtures below $w=0.5$ which was confirmed by contact preparations for polarized light microscopy (PLM) under ambient conditions. These showed a quick formation of a myelin like phase near the borderline that did not change within hours (Fig. 3a). We interpret these findings with the formation of extended lamellae. These impede or significantly retard further dilution but can swell. Indeed SAXS peaks in the ultra-small angle region for both systems correspond to 100-200 nm large objects (shown for 3a, Fig. S2 $\dagger$ ). Such a huge spacing of swollen BA based lamellae is not unusual ${ }^{18}$ and has also been reported for dilute ASA-water systems. ${ }^{19}$ Upon standing over several months 3a $(w=0.54)$ macroscopically phase separates into an isotropic phase (no birefringence, no peaks in SAXS) beneath a lamellar phase as indicated by three harmonic peaks with a corresponding repeat distance of $7.9 \mathrm{~nm}$ (PLM: Fig. 3b, SAXS:
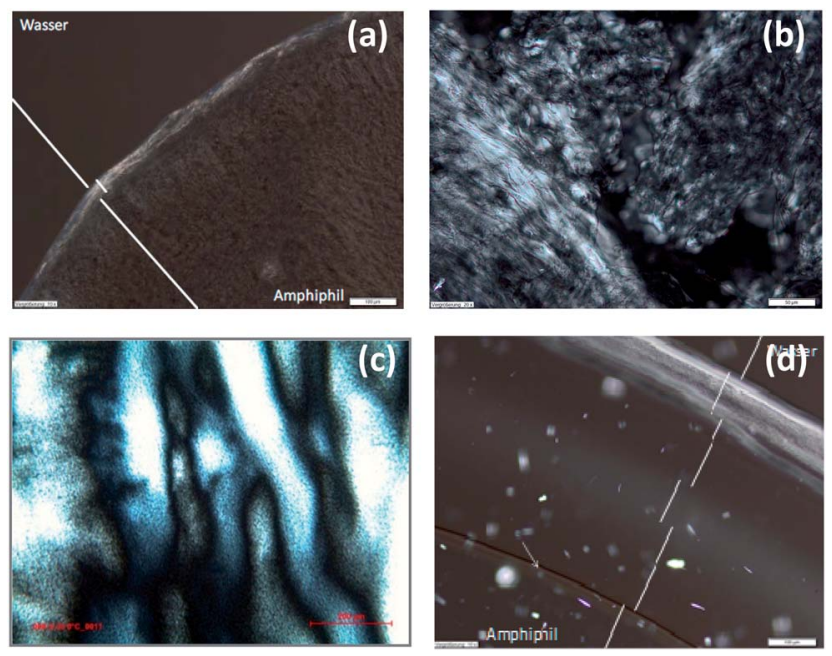

Fig. 3 (a) Contact preparation of 3a showing a myeline like interphase diagonally demarcating water (to the left) and the amphiphile (scale bar $100 \mu \mathrm{m}),(\mathrm{b})$ PLM picture of the phase separated lamellar phase of $3 \mathrm{a}(\mathrm{w}$ $=0.54)$ (scale bar $50 \mu \mathrm{m})$, (c) PLM picture of $3 \mathrm{c}$ at $0{ }^{\circ} \mathrm{C}(w=0.33)$ (scale bar $200 \mu \mathrm{m})$, (d) contact preparation between $3 f(w=0.82)$ showing batonnets and water (upper left) (scale bar $100 \mu \mathrm{m}$ ).
Fig. 4a). These phases consist of distinct different PES-BA species of 3a. While a low molecular weight fraction builds the lamellar phase, the isotropic contains polymeric amphiphiles (Fig. 2d).

For ASA terminated PES macroscopic homogeneous and storage stable dispersions could be obtained down to $w \sim 0.3$. Apart from peculiarities reflecting an impact of the nature of the alkenyl side group all PES-BA 3c-3g show ordering somewhere within the parameter space defined by temperature $\left(0-80{ }^{\circ} \mathrm{C}\right)$ and composition $(0.3<w \leq 0.6)$ according to SAXS reflections (listed, Table $\mathrm{S} 2 \dagger$ ) and birefringence textures. Except for $\mathbf{3 g}$ these peaks shift to lower $q$ values with decreasing concentration indicating larger distances or objects. Besides these reflections humps appear which will be discussed below.

Thus PES-BA 3c (C8 chains) at $w=0.33$ shows increased ordering upon cooling from $20{ }^{\circ} \mathrm{C}$ to $5{ }^{\circ} \mathrm{C}$ where the hump transforms into a sharp peak (Fig. 4b) while the Schlieren-like birefringence, typical for nematic ordering, becomes more intense (Fig. 3c). Both vanish at higher temperatures, shown for SAXS at $70{ }^{\circ} \mathrm{C}$. A similar, less pronounced temperature dependence was found with PES-BA $3 \mathbf{f}$ (tetrapropenyl C12) at $w=0.49$ where the hump only appears upon cooling. With increasing concentration $\mathbf{3 f}$ forms batonnets as was shown with PLM in a contact preparation (Fig. 3d).
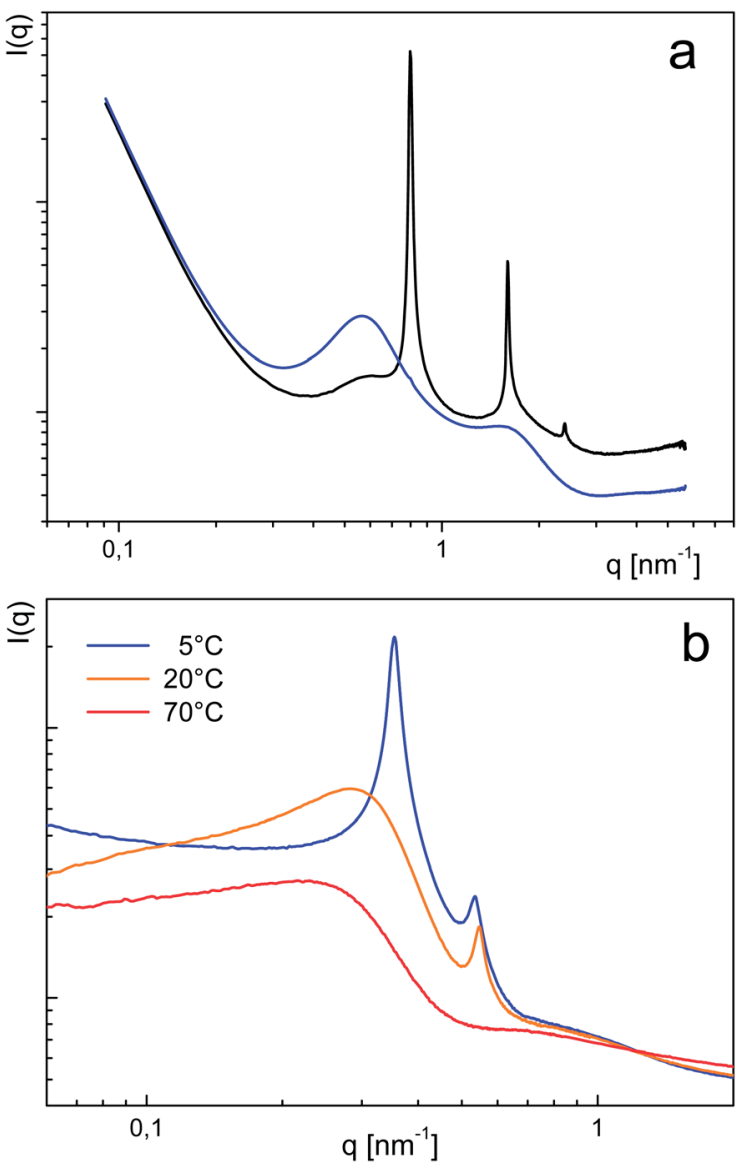

Fig. 4 SAXS curves obtained on (a) phase separated (3a) $w=0.54$ into a lamellar (black, $d=7.88 \mathrm{~nm}$ ) and an isotropic phase (blue) and (b) peaks of (3c) $w=0.33$ at $5{ }^{\circ} \mathrm{C}, 20^{\circ} \mathrm{C}$ and $70{ }^{\circ} \mathrm{C}$. 
The isomeric 3e (branched C12 chains) does not show any birefringence between $0.34 \leq w \leq 0.47$ below $50{ }^{\circ} \mathrm{C}$. This might be due to homogeneous alignment as SAXS displays both types of reflections. Above $50{ }^{\circ} \mathrm{C}$ birefringence without texture of an ordered isotropic, e.g. bicontinuous, phase appears. At lower concentration $(w=0.31$, not shown $)$ this was found within huge isolated domains of a non-birefringent liquid.

Increasing both the length and the non-uniformity of the aliphatic chains in $\mathbf{3 g}$ (branched C16 and C18 chains) leads to aqueous compositions ( $w=0.5-0.6)$ that show anisotropy in SAXS (Fig 5b). The birefringence texture of shear banded oily streaks persists up to $50{ }^{\circ} \mathrm{C}$ and is evocative of a lamellar phase (Fig. 5a). However the correlation length is rather limited regarding missing harmonics.

In conjunction with the morphological variety of mesophases formed by bola-amphiphiles in binary systems with water over vast concentration and temperature ranges ${ }^{8}$ the clarification of the self-assembly of PES-BA $3 \mathbf{a}$ to $\mathbf{3 g}$ during the manufacturing procedure remains a matter of further studies, e.g. in situ SAXS measurements. However with regard to reported bi-layered membrane formation in concentrated C16-ASA solution ${ }^{19}$ and the shift from vesicle to disk formation in bolaamphiphile water systems with increasing temperature from $20{ }^{\circ} \mathrm{C}$ to $60^{\circ} \mathrm{C}$ (ref. 12 ) we assume that the PES-BA tend to adopt layered assemblies in the course of the dilution process. This is corroborated by our findings on the dilution of succinic and maleic ester bearing PES-BA 3a and $\mathbf{3 b}$ resp. (vide supra), distinct peaks ( $1^{\text {st }}$ and $2^{\text {nd }}$ harmonic) in concentrated phases of TMA terminated PES-BA $3 \mathbf{h}$ (Table S2, $\dagger$ n.b.: double ionic strength) as well as indications for lamellae formed by ASA terminated PES-BA 3g. Contrary to the succinic (and maleic) terminated counterparts, the latter can be further diluted and does not phase separate. This suggests either the presence of less extended or porous lamellae and (or) intermolecular stabilization via hydrophobic attraction of the terminal hydrophobic side chains.

However in the case of ASA head groups that bear shorter (linear or branched) chain stabilization seems to favor finite anisotropic objects like rods, disks or ribbons that tend to nematic ordering ( $c f$. 3c, 3f). Indeed cryo-AFM imaging of PESBA 3c $(w=0.4)$ (Fig. 6) and $3 \mathbf{f}(w=0.49)$ (Fig. S1†) suggests preferentially aligned anisotropic objects of dimensions and

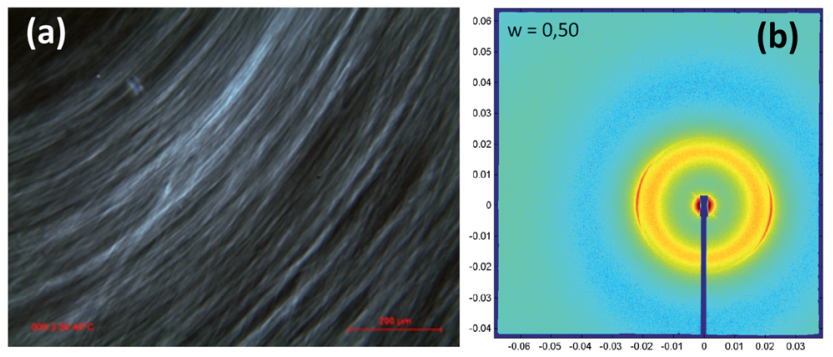

Fig. 5 (a) Birefringence of $3 \mathrm{~g}(w=0.5)$ at $40^{\circ} \mathrm{C}$ (scale bar $200 \mu \mathrm{m}$ ) and (b) 2D SAXS pattern of the same phase at $20^{\circ} \mathrm{C}$, showing an oriented mesophase with respect to the peak positions at $q=0.46 \mathrm{~nm}^{-1}$.

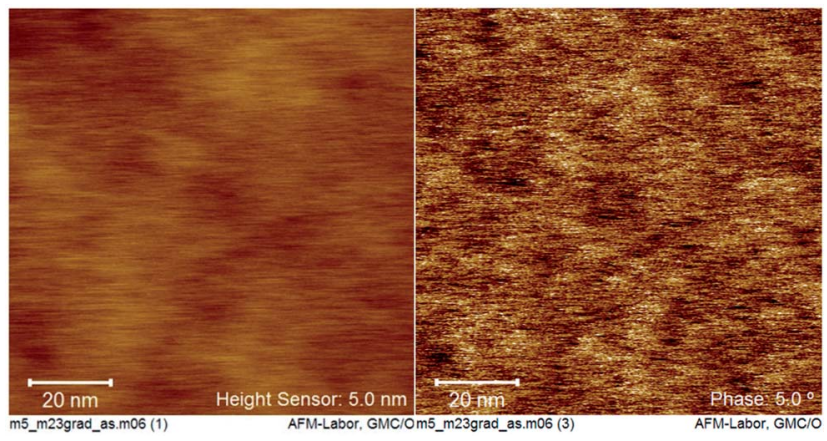

Fig. 6 Cryo-AFM images (topography left, phase contrast right) of $3 \mathrm{c}$ $(w=0.4)$ reveal aligned domains with dimensions and spacings of the order of 10 to $20 \mathrm{~nm}$ respectively, resembling the nematic order.

spacing that roughly correspond to observed humps and peaks in SAXS (appr. 10-20 nm).

These humps might reflect a 'correlation hole effect' rather than an ordering phenomenon: only by using a high flux X-ray source Cortese et al. observed broad reflections in bolamaphile dispersions and attributed them to the distance between electron richer heads attached to the aliphatic spacer. ${ }^{20}$ However with regard to the esterification process of PES (vide supra) this interpretation would imply unlikely high proportions of high molecular weight species, e.g. 2c capped HPA : DFA : HBA = $3: 3: 7$ (18.3 nm, Table $\mathrm{S} 1 \dagger)$, that could cause humps around 20 $\mathrm{nm}$. As their positions shift to lower $q$ values with decreasing $w$ values (Table S2†) we assume that they reflect disordered dispersion states of discrete objects consisting of assembled bola-amphiphiles. This is in line with the hump to peak transition in the scattering curves of $3 \mathbf{c}(w=0.33)$ during cooling (Fig. 4b).

Such objects may be formed by co-assembly of polymeric and diester based bola-amphiphiles ( $c f$. composition of PES) while anisotropy arises from their different molecular dimensions of $\sim 5$ to $10 \mathrm{~nm}$ versus $\sim 2 \mathrm{~nm}$ respectively ( $c f$. Tables $\mathrm{S} 1$ and $\mathrm{S} 2, \uparrow$ peak positions). There is a pronounced shift of the peaks around $7 \mathrm{~nm}$ to values around $10 \mathrm{~nm}$ with decreasing concentration for all ASA bearing systems. This shift is much less for higher $w$ values of $\mathbf{3 h}$. We interpret this by a variable degree of chain elongation of longer, flexible amphiphiles, especially DFA comprising ones, in response to favorable charge separation as a function of the number of ionic groups (higher for $\mathbf{3 h}$ ) and the degree of dissociation which should increase upon dilution. For the succinic ester and ASA based systems accommodation of oligomers with intermediate molecular weight and dimensions has to be considered and discussed in the context of lower degrees of carboxylic acid neutralization. Indeed considerable amounts of amine evaporated in the course of sample preparation (Table S2 $\dagger$ ). Consequently, as a function of water content, varying amounts of non-neutralized acid groups of shorter bola-amphiphiles could be embedded within the hydrophobic core of the assemblies (Fig. 7). Such an accommodation seems reasonable in the light of similar findings reported for glycerol terminated asymmetric bola-amphiphiles., ${ }^{\mathbf{8} 21}$ 


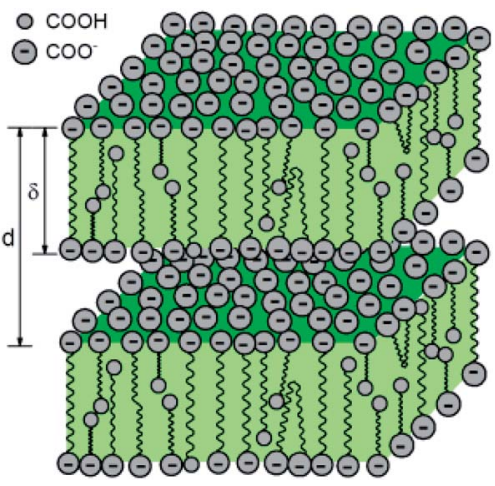

Fig. 7 Assembly model of polymeric bola-amphiphiles of different length, symmetry (carboxylic acid, carboxylate) and conformation (stretched, bended). Dimensions are not to scale.

The different behaviors of succinic and alkenyl succinic bearing amphiphiles reveal a twofold role of hydrophobic side chains neighboring the polar head group: (i) stabilization of assemblies of different species and (ii) packing frustration that impedes the formation of extended lamellae.

To summarize the results we have shown that ordered mesophases can be obtained from an ill-defined system of bolaamphiphiles comprising monomeric and polymeric species and that the presence and the nature of terminal hydrophobic side groups have a distinct impact on the assembly and thus the degree of order of the obtained dispersions. In the following chapter ordering phenomena of ASA based HBA are described in more detail.

\section{Mesophases from monomeric diester bola-amphiphiles}

Hydrogenated bisphenol-A alone - despite being rather bulky and hydrophobic - apparently fails to provide a suitable hydrophobic spacer for the diester $\mathbf{4 a}$ (succinic) and $\mathbf{4 h}$ (trimellitic). The corresponding ammonium salts do not show any birefringence in contact preparations and in the bulk $\mathbf{4 h}$ quickly dissolves as a bola electrolyte (not shown). In order to shed light on the assembly of ASA terminated PES-BA, reference compounds $4 \mathbf{c}$ (linear C8), 4d (linear C12) and 4e (branched C12) have been prepared and their aqueous mesophases have been studied. Apart from their uniform molecular weights these compounds, like their polymeric counterparts, consist of molecular mixtures regarding isomerism of the diol, the alkenyl chain and its position relative to the succinic ester group (vide infra).

Although heating would be unnecessary with regard to low viscosities compared to the concentrated PES-BA, the neutralized diester was diluted with water at temperatures above $60{ }^{\circ} \mathrm{C}$. Thus homogeneous, transparent and storage stable dispersions were obtained either in the form of gels or viscous fluids in the concentration range $0.3 \leq w \leq 0.7$. In contrast, dilution of $4 \mathbf{e}$ at room temperature yields heterogeneous mixtures of a coarsely dispersed white waxy solid indicating metastable regions in the phase diagram of this system.

Structural analysis of the aqueous phases of $4 \mathbf{c}, \mathbf{4 d}$ and $4 \mathbf{e}$ reveals a common feature in SAXS: two more or less pronounced peaks at positions in line with lamellae of decreased spacing with increasing bola-amphiphile contents as shown for $\mathbf{4 c}$ (Fig. 8). At the lowest concentration the main reflex is rather broad and the second peak barely visible as a step while at $w=$ 0.4 faint peaks of inverted intensities are found (besides a weak $3^{\text {rd }}$ harmonic). For higher concentrations peak intensities rise. Similar sets of peaks are found for the mesophases of $4 \mathbf{d}$ and $4 \mathbf{e}$ (Fig. S3 and Table S4 $\dagger$ ). However for the linear C12 chains these are only present at higher bola-amphiphile contents $(w \geq 0.6)$. Compared to the corresponding $\mathrm{C} 8$ based phase the repeat distance increases about $0.63 \mathrm{~nm}(w=0.7)$, which nearly matches twice the length of an all-trans $\mathrm{C} 4$ increment $(0.312$ $\mathrm{nm})$. At lower concentrations broad humps indicate poor ordering. Intriguingly the branched C12 groups impede ordering at the highest concentration while the abnormal peak intensities described above for $4 \mathbf{c}(w=0.4)$ are shifted to a concentration of $w=0.5$. Compared to the other amphiphiles at $w=0.7$ the differences of the peak (or hump) positions translate into roughly half the distance found for the comparison between $\mathbf{4 c}$ and $\mathbf{4 d}$, specifically $0.34 \mathrm{~nm}$ larger than in $\mathbf{4 c}$ and $0.29 \mathrm{~nm}$ smaller than in $\mathbf{4 d}$ (Table S4†). This would be in line with a less extensive space filling of the branched chains in $4 \mathbf{e}$. Although plausible with regard to molecular extensions the difference between repeat distances in $\mathbf{4 c}$ and $\mathbf{4 d}$ at high $w$ values can hardly be explained by a typical bola-amphiphile assembly. Such lamellar stacking between an aligned hydrophobic spacer and water with the polar heads forming the interface implies that the alkenyl chains should oriented inbound rather than into the water phase. However a bilayer type assembly of molecules with their two hydrophobic side chains being oriented asymmetric and two elongated ones forming the hydrophobic core of a lamella could explain the findings.

All phases at $w=0.3$ except for 4c (Fig. 9a) and those at higher $w$ values which only show humps in SAXS do not display any birefringence. Remarkably those which cause faint and

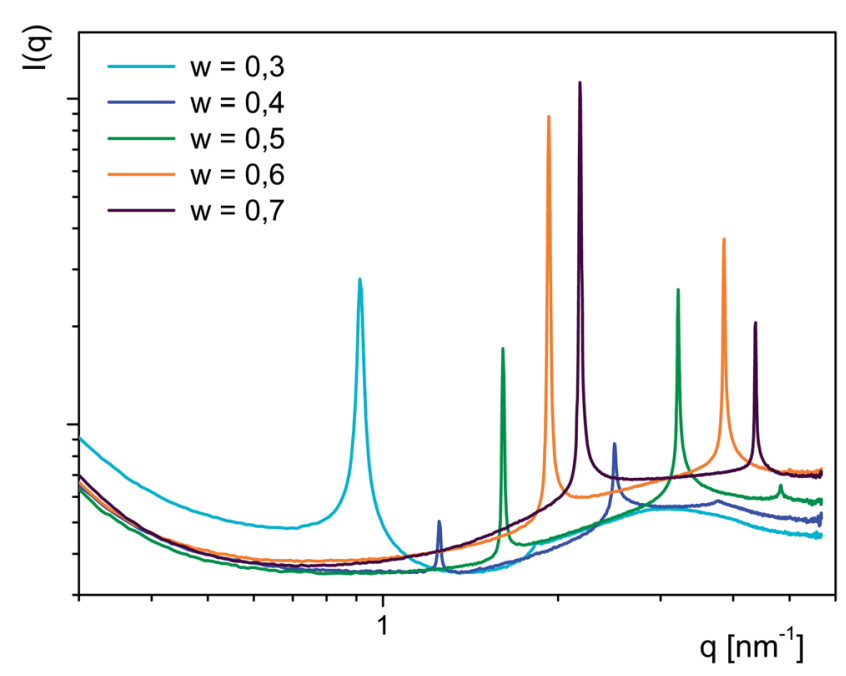

Fig. 8 SAXS curves from phases of $4 \mathrm{c}$ at $0.3<w>0.7$ and $20^{\circ} \mathrm{C}$. Peaks shift to higher $q$ values with increasing $w$. 

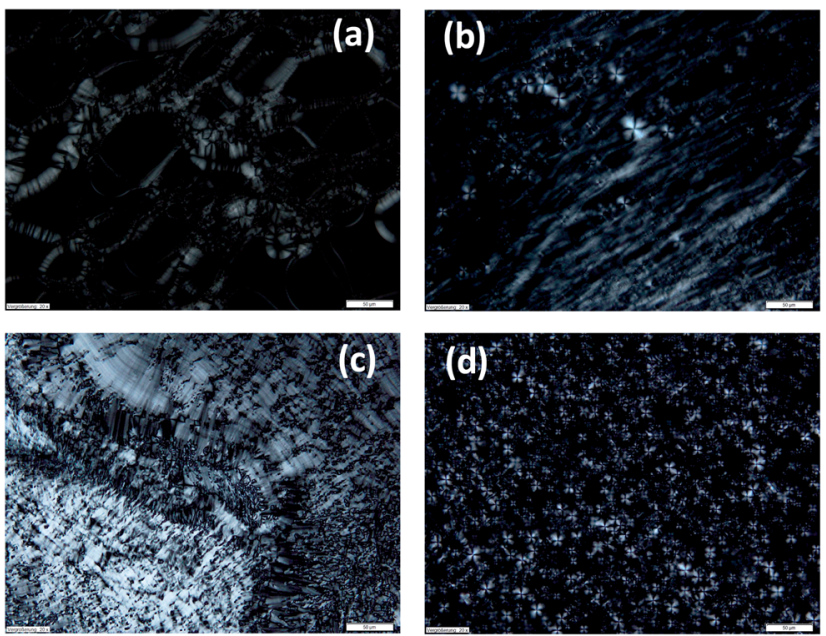

Fig. 9 PLM pictures of (a) $4 c w=0.3$, (b) $4 c w=0.4$, (c) the same as in (b) after shearing the cover slip, and (d) $4 \mathrm{e} w=0.5$. All scale bars $50 \mu \mathrm{m}$

unusual SAXS peak ratios exhibit distinct shear dependent birefringence with oily streaks transforming into a marble texture ( $4 \mathbf{c} w=0.4$ Fig. $9 \mathrm{~b}$ and c) or into the dominant Maltese crosses (4e $w=0.5$ Fig. 9 d) respectively. Such behaviour is evocative of lamellar polymorphism observed in several bilayered assemblies of two- and multi-component aqueous detergent mixtures. Thus the evolving texture of $4 \mathbf{e}(w=0.5)^{22}$ could be indicative for a transition of a lamellar phase $\left(\mathrm{L}_{\mathrm{a}}\right)$ into a multi-lamellar vesicle $\left(\mathrm{L}_{\mathrm{a} 1}\right)$ phase. ${ }^{23}$ Indeed a cryo-SEM image of this mesophase reveals a lamellar morphology comprising domains with a distinct curvature (Fig. S5 $†$ ). For the texture change of $4 \mathbf{c}(w=0.4)$ polymorphism between an isotropic sponge $\left(\mathrm{L}_{3}\right)$ and a lamellar $\left(\mathrm{L}_{\alpha}\right)$ or mesh phase respectively is reasonable. ${ }^{24}$ The former is known to readily align under an external force into the latter, both sharing scale invariance ${ }^{25}$ and form in concentrated regimes and persist into dilute regions. ${ }^{26}$

These observations are corroborated by ${ }^{2} \mathrm{H}-\mathrm{NMR}$ experiments on corresponding samples, dissolved in $\mathrm{D}_{2} \mathrm{O}$ on an equimolar basis with regard to the $\mathrm{H}_{2} \mathrm{O}$ based counterparts. The extent of the ${ }^{2} \mathrm{H}$ quadrupole splitting depends on the degree of anisotropy at the amphiphile water interface and thus indicates the symmetry of the mesophase as well as the sample heterogeneity in the case of coexisting mesophases of different space groups. ${ }^{27}$ Curves of the mesophases of $\mathbf{4 c}$ (Fig. 10) show biphasic samples for $w=0.3$ (main portion isotropic, compared with birefringence, Fig. 9a) and $w=0.4$ (a set of peaks and shoulders respectively). At higher concentrations the anisotropy of the interface and thus the splitting increase to values typical for lamellar interfaces (peak separation $\sim 1.700 \mathrm{~Hz}$ ) ${ }^{28}$ However the smooth "filling" between the peaks indicates the presence of more isotropic parts in these interfaces. Similar behaviour is found for $\mathbf{4 d}$ and $4 \mathbf{e}$ (Fig. S4 $\dagger$ ). Their less ordered phases (humps in SAXS curves, vide supra) do not show quadrupole splittings while for the ordered concentrated phases of $4 \mathbf{d}$ the splitting equals that of $\mathbf{4 c}$. For the branched $\mathrm{C} 12$ bearing bola-

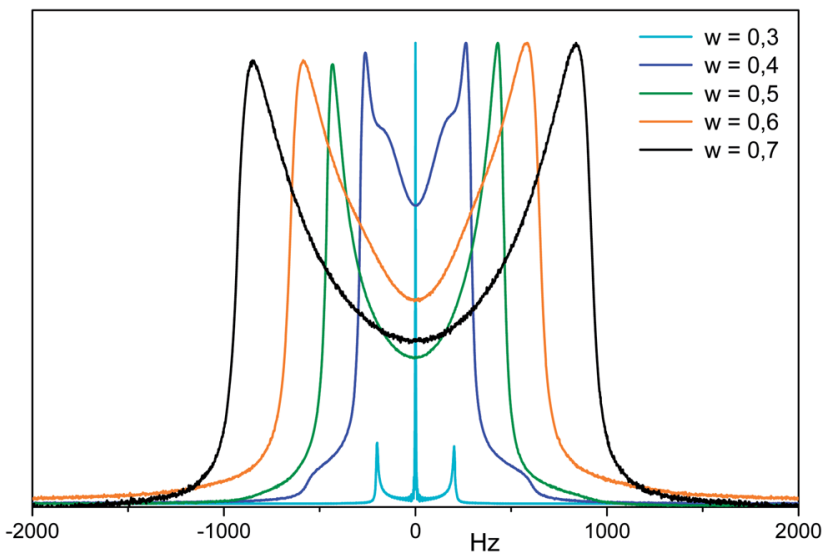

Fig. $10{ }^{2} \mathrm{H}$-NMR of $4 \mathrm{c}$ in $\mathrm{D}_{2} \mathrm{O}$ showing deuterium quadrupole splittings increasing with concentration ( $w$-values are rounded, see Experimental part). At $w=0.3$ (compare OPM picture, Fig. 9a) and $w=$ 0.4 , samples are biphasic.

amphiphile 4e maximum splitting is much less $(644 \mathrm{~Hz})$ compared to that of both other systems.

While the structural analysis of the ordered phases reveals isotropic features of a lamellar morphology rheological properties of these phases accentuate the isotropic aspect. Indeed amplitude sweeps reveal a distinct gel character of $\mathbf{4 c}$ over the whole concentration range with $G^{\prime}$ exceeding $G^{\prime \prime}$ by factors of 6 to 15 and yield points between 5 and $45 \mathrm{~Pa}$. $\|$ This could be best described with a percolating, force transducing, bicontinuous morphology. The ordered phases of $4 \mathbf{d}(w \geq 0.6)$ and of $4 \mathbf{e}(w<$ $0.6)$ show a similar behaviour while the less ordered phases (humps in SAXS) of both $\mathbf{4 d}(w<0.6)$ and $\mathbf{4 e}(w>0.6)$ are viscous fluids. This is exemplary shown for the phases at $w=0.5$ (Fig. 11) and leaves $4 \mathbf{e}$ at $w=0.6$ as an exceptional dispersion where order and viscous behaviour coincide.

For all ordered dispersions of $4 \mathbf{e}(0.3<w<0.6)$ elasticity and viscosity considerably decline upon heating in the range of $35-$ $60{ }^{\circ} \mathrm{C}$, with higher concentrated phases becoming fluid more quickly (Fig. S6a $\dagger$ ). This is accompanied by a loss of the anisotropic structure, revealed by quadrupole splitting (Fig. S6b†). Noteworthy inverse behaviour takes place with $4 \mathbf{d}$ at $w=0.5$. Being isotropic at room temperature, this system forms a coexisting anisotropic part upon heating (Fig. S7b†). This transforms the viscous liquid into an elastic dispersion at $50{ }^{\circ} \mathrm{C}$ (Fig. S7a †). All dispersions of $\mathbf{4 c}$ and the ordered phases of $\mathbf{4 d}$ retain moduli at least up to $80^{\circ} \mathrm{C}$ or $90{ }^{\circ} \mathrm{C}(4 \mathrm{c} w=0.5,4 \mathbf{d} w \geq$ $0.6)$ respectively (Fig. S8a and S7a†). For $4 \mathbf{d}(w=0.7)$ an isotropic fraction is formed around the yield point which dominates in the fluid region (Fig. S7b † ) while only slight

|| Measured at $1 \mathrm{~Hz}$. frequency sweeps of $\mathbf{4 c}$ and $\mathbf{4 e}(w=0.4)$ as well as $\mathbf{4 d}(w=0.5$ and 0.6) measured with an amplitude of $0.1 \mathrm{mrad}$ are shown in Fig. S12. Between 1 and $100 \mathrm{~Hz}$ moduli steadily increase, the loss moduli of the hydrogels approaching the storage moduli at the high frequency end. For the viscous liquid $4 \mathbf{d}(w=0.5)$ exponentially increasing $G^{\prime}$ narrows the gap between moduli at higher frequency. The typical range of shear stress covered by the sweeps was in the range of 0.4 and $18 \mathrm{~Pa}$ for the hydrogels and 10 to $24 \mathrm{~Pa}$ for the viscous liquid of $\mathbf{4 d}(w=0.5)$. 


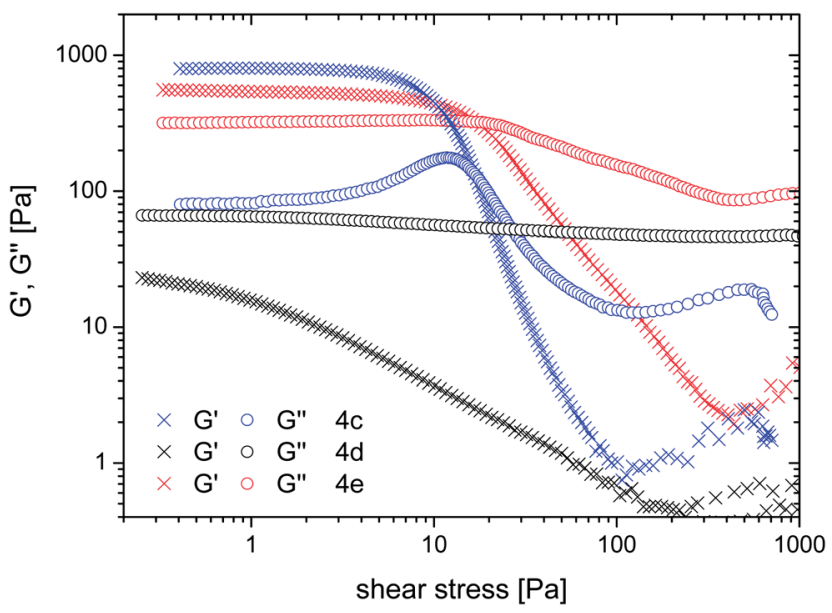

Fig. 11 Amplitude sweeps performed at $20^{\circ} \mathrm{C}$ and $1 \mathrm{~Hz}$ on the mesophases comprising of distinct hydrogel $4 \mathrm{c}$ (blue), viscous fluid $4 \mathrm{~d}$ (black) and hydrogel $4 \mathrm{e}$ (red) at $w=0.5$. Storage modulus $G^{\prime}$ (crosses), loss modulus $G^{\prime \prime}$ (circles).

changes in the quadrupole splitting of $\mathbf{4 c}$ based dispersions were observed until $80{ }^{\circ} \mathrm{C}$ like the merger of the coexisting curvatures at $w=0.4$ ( $c f$. Fig. 10) into one anisotropic phase around $60{ }^{\circ} \mathrm{C}$ (Fig. S8b†).

To summarize the experimental results we have shown that HBA based bola-amphiphiles with C8-C12 ASA head groups self-assemble in water into anisotropic structures resembling mesh and coexistent bicontinuous phases. With regard to the terminal hydrophobic side group the linear C12 system fails to maintain ordered assemblies with increasing dilution whereas non-uniform, bulky groups of the branched C12 system impede ordering at high concentration and the formation of temperature stable structures in dilution. In contrast linear C8 groups allow for ordered structures over the entire range of covered concentrations and temperatures.

With regard to the polymorphism we suggest that molecular diversity of the diester bola-amphiphile most probably impedes the formation of different well-defined, e.g. lamellar assemblies. We calculated a possible composition of $\mathbf{4 c}$ (the same would apply to $4 \mathbf{d}$ ) on the basis of analytical results regarding isomerism of educts and reaction products. As outlined in the ESI $\dagger$ HBA consists of five species. Apart from two minor unassigned monools the diol $(w=0.97)$ comprises a trans,trans $(w=0.50)$, a cis,trans $(w=0.42)$ and a cis,cis $(w=0.08)$ isomer. ASA 2c consists of trans- and cis-configured double bonds in the unsaturated chain in a $4: 1$ ratio. Anhydride addition yields two different succinic ester groups with alkenyl chains either in the $\alpha$ - or $\beta$-position $(\alpha / \beta=0.61)$. Lacking further information concerning symmetry with respect to isomeric head groups in $\mathbf{4 c}(\mathbf{d})$ we assume independent probabilities and ignore the monool fraction. Thus 35 different species in proportions between 21.73 and $0.97 \mathrm{~mol} \%$ (Table S3 $\dagger$ ) are obtained. On this basis it is reasonable that bola-amphiphiles of different shapes (e.g. cis,cis vs. trans, trans) and varying distances between the hydrophobic chains (e.g. $\alpha, \alpha$ - vs. $\beta, \beta)$ can be allocated to sites of different curvature in order to minimize packing frustration. Thus molecular diversity induces polymorphism of a few rather similar structures over a broad concentration range, contrary to molecular uniform systems that usually display morphological plurality with varying water content. ${ }^{29}$ Indeed molecular simulations of $4 \mathbf{c}$ in water in the range of $0.3 \leq w \leq 0.6$ yield anisotropic morphologies like biased bicontinuous phases (shown for $w=0.3$, Fig. 12) and porous lamellae. It is noteworthy that although slight changes in isomer compositions can generate qualitative changes in the morphology, all of them resemble anisotropic meshes or bicontinuous phases. These results confirm the experimental findings and support our interpretation.

Thermal stability as well as the order at very high concentration decreases in the case of branched side groups while
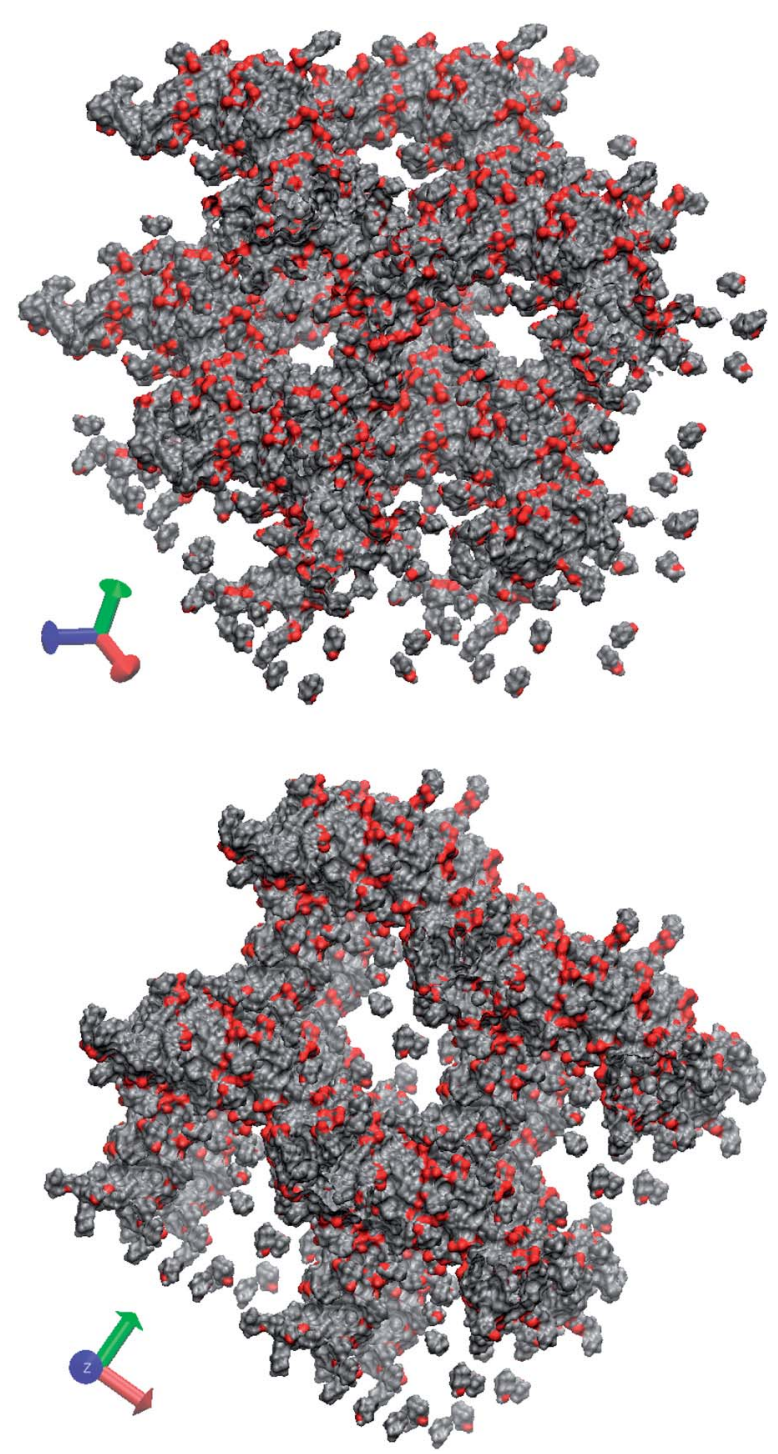

Fig. 12 Simulated bicontinuous morphology of $4 \mathrm{c}$ in water $(w=0.3)$ after $100 \mathrm{~ns}$ at $20^{\circ} \mathrm{C}$. Box size 16.000 atoms comprising 34 molecules of $4 \mathrm{c}$, taking into account the 15 most prevalent isomers (see text and Table S3†). Only the molecules of $4 \mathrm{c}$ and the ammonium ions are shown (red $=$ oxygen atoms) while the box is periodically repeated once in each direction $(x, y, z)$ for clarity. 
longer linear chains impede ordering at lower concentrations. The former is intuitively understood regarding the bulkiness of the hydrophobic moieties. For the longer chains most probably intermolecular hydrophobic attractions between just a fraction of differently shaped isomers induce rupture of the percolating assembly as it is evidenced by the fluid nature of the more dilute dispersions.

\section{Bola-amphiphiles, bola-electrolytes and non-bola- amphiphiles}

As mentioned in the preceding chapter compounds $\mathbf{4 a}$ and $\mathbf{4 h}$ do not form ordered assemblies in water** as do the ASA based analogues. With respect to the latter we were interested in the question at which point with respect to the spacer length the ordering would be changed or impeded: where does the bola-amphiphile transform into a Gemini surfactant or a bolaelectrolyte respectively? We synthesized the diester from 1,4-bis(hydroxymethyl)cyclohexane (cis : trans $=0.43$ ) with $2 \mathrm{a}$, 2c and 2e respectively in order to maintain the bulkiness and rigidity of HBA. However all dispersions of the neutralized diacids $\mathbf{5 a}, \mathbf{5 c}$ and $\mathbf{5 e}$ are transparent low viscous fluids that do not display any birefringence in OPM, contrary to the hydrolyzed and neutralized ASA 2c which forms well-defined mesophases in water (Fig. S9†). Thus a shorter yet rigid spacer forces ASA based diester to dissolve as bola-electrolytes.

In the preceding section the pronounced tendency of $4 \mathbf{c}$ to form hydrogels of distinct temperature stability was shown, while PES-BA 3c assembles into nanosized, nematic aligned objects. We expected a pronounced gel character for dispersions of higher molecular weight polyester that comprise angular chains or a branched architecture like PES-NB, a chain extended PES via TMA (Fig. 1). The hydroxyl terminated polyester bears mid chain carboxylic acids. Dilution of the molten neutralized PES at $80{ }^{\circ} \mathrm{C}$ yields homogeneous, translucent hydrogels in the concentration range $0.7 \geq w \geq 0$. With $w=$ 0.35 a freshly prepared dispersion displays Schlieren texture of a nematic ordering which changes into featureless birefringencet† within a few days (Fig. 13). Cryo-TEM images, taken after several weeks, reveal worm like patterns of truncated bicontinuous phases (Fig. 14). After 26 months under ambient conditions the gel still manifests its undulated surface (Fig. S10 $\dagger$ ) and tangibly oscillates in response to manual knocking against the container. The distinct time dependency of the ordering that produces an elastic solid with a pronounced yield point prompted us to study the rheological development of the dispersion in the temperature range of 10 to $60{ }^{\circ} \mathrm{C}$. Heating causes an increase of the loss modulus $\left(G^{\prime \prime}\right)$ until $30^{\circ} \mathrm{C}$ followed by a decrease of both moduli until, at $60{ }^{\circ} \mathrm{C}, G^{\prime \prime}$ dominates the storage modulus $\left(G^{\prime}\right)$. Comparing a freshly prepared with an aged sample the gel fluid transition starts on a much higher viscosity level in the latter (not shown). Upon cooling of the heated sample the material follows simple Arrhenius behavior down to $30{ }^{\circ} \mathrm{C}$. Below that temperature $G^{\prime}$ increases further

** Missing birefringence, but dried 4a displays textures of crystals.

$\dagger$ Macroscopic the sample can easily be sliced into extended flakes. while $G^{\prime \prime}$ decreases, ending with an elastic solid at $10{ }^{\circ} \mathrm{C}$ (Fig. S11 $\dagger$ ). The pronounced inertia of PES-NB is reflected by the slow development of the viscoelastic components over time at higher temperatures (Fig. 15).
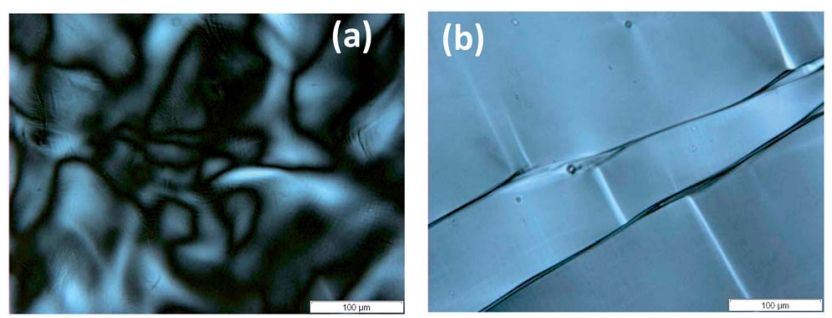

Fig. 13 PLM pictures of PES-NB in water $(w=0.35)$ (a) freshly prepared and (b) after 7d at ambient temperature.
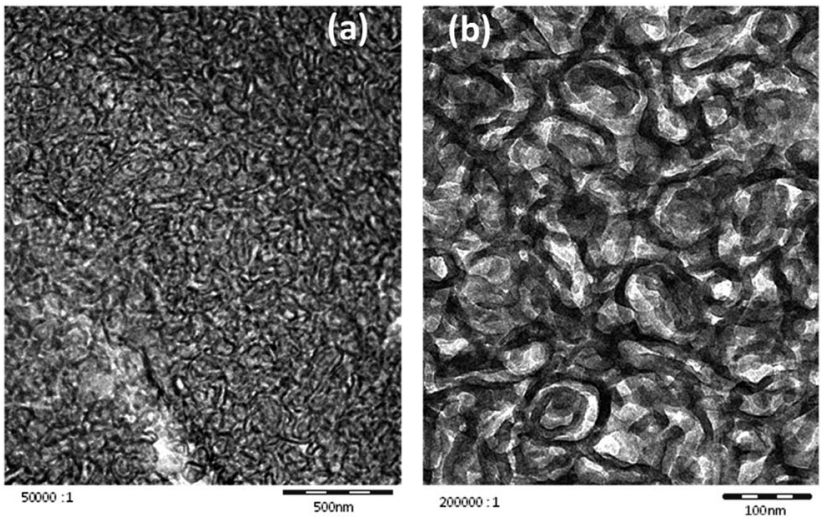

Fig. 14 Cryo-TEM images of an aged dispersion of PES-NB in water $(w=0.35)$ showing a truncated bicontinuous morphology (a) cross sectioned (scale bar $500 \mathrm{~nm}$ ), (b) in projection (scale bar $100 \mathrm{~nm}$ ).

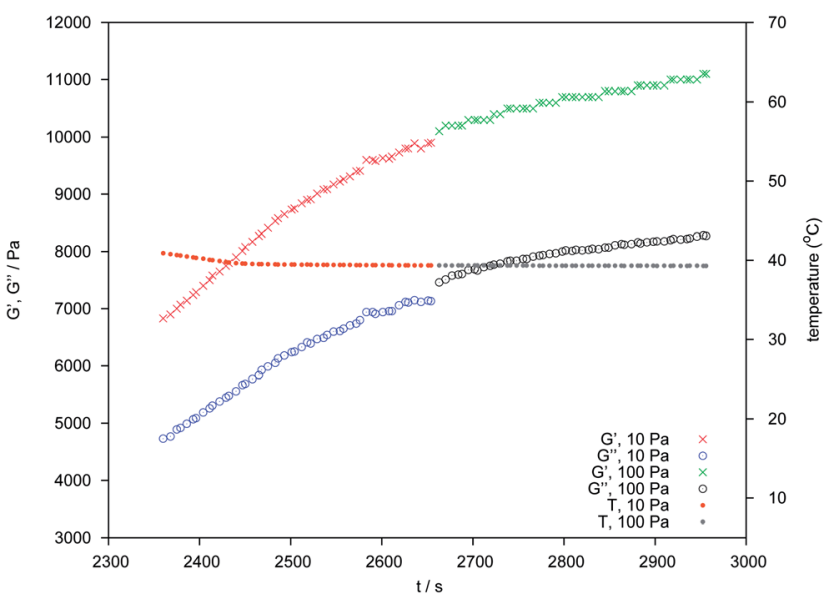

Fig. 15 Oscillating shear rheology on PES-NB in water $(w=0.35)$ at $40{ }^{\circ} \mathrm{C}$ taken during the cooling phase of a preheated $\left(60{ }^{\circ} \mathrm{C}\right.$ max.) sample. The evolution of loss $\left(G^{\prime \prime}\right.$, circles) and storage $\left(G^{\prime}\right.$, crosses) modulus in the time domain was followed at two different shear stresses (10 Pa, blue and red and $100 \mathrm{~Pa}$, green and black). 


\section{Conclusions}

The present study describes an economic and scalable approach to anisotropic liquids and hydrogels making use of a novel bola-amphiphile motif consisting of alkenyl succinic ester terminated hydrophobic di- and polyester based spacers. We have shown that a remarkable degree of order can be obtained from ill-defined mesogens which are based on technical grade raw materials consisting of numerous isomers as well as a plurality in molecular weights regarding polymer comprising reaction mixtures. With regard to the latter we have shown that terminal hydrophobic side groups stabilize assemblies formed from species of different molecular weight and size. Such anisotropic nanosized objects display nematic or lamellar like ordering depending on the length of the terminal side chains.

Monomeric diester bola-amphiphiles from C8 to C12 alkenyl succinic ester attached to hydrogenated bisphenol-A as a rigid spacer yield ordered dispersions. Such hydrogels form over the most extended concentration and temperature range in the case of C8 side chains. These phases can best be understood as polymorphism between porous lamellae (mesh) and a $\mathrm{L}_{3}$ sponge phase. The plurality of isomeric species with different shape and aliphatic chain position allows for the allocation of different curvatures of the water organic interface, thus leading to scale invariance and nearly constant elastic response to external force. Longer as well as bulkier groups narrow the concentration range for the ordered phases and, in the case of branched chains, their temperature stability.

It will be the matter of further studies to unravel in more detail the mesogen behaviour of individual isomers of the ASAHBA family with regard to the spacer as well as the different side groups.

Regarding both the economic and sustainable feedstock with respect to alkenyl succinic anhydrides and dimer fatty acid as well as the simple synthesis and manufacturing processes, and taking into account comparatively low amounts of polar groups, the authors anticipate that the presented polymer based approach will pave the way for ordered liquids as a formulation basis in the development of anisotropic materials for large scale applications like functional films, coatings or adhesives. Thus it is conceivable to make use of surface induced ordering effects be it macroscopic or internal ones like layered nano particles, process related small shear flow or intrinsically replicating order phenomena in passing through the systems phase diagram in the course of water evaporation.

However careful validation of technical grade raw materials and possibly adaptation of process parameters in the course of the scale-up procedure are required for the sake of quality control.

\section{Acknowledgements}

The authors would like to thank Svetlana Guriyanova ${ }^{1}$ (cryoAFM), Volodymyr Boyko ${ }^{1}$ (SAXS), Thomas Frechen ${ }^{1}$ (cryo-TEM, SEM), Elke Austrup ${ }^{2}$ (PES synthesis), Martina Moellers ${ }^{2}$ (PLM), Klaus Brockkötter ${ }^{2}\left({ }^{2} \mathrm{H}-\mathrm{NMR}\right)$, Julia Uckelmann ${ }^{2}$ (rheology), Alex
Oliver $^{3}$ and Charl Faul ${ }^{3}$ (starting from PES, $1^{\text {st }}$ trials to synthesize and to disperse the systems $\mathbf{3 a}, \mathbf{3 d}, \mathbf{3 h}$, in the frame of a summer project in 2011), Reinhard Doetzer ${ }^{1}$ for fruitful discussions in the context of the HBA isomer assignment. ${ }^{1} \mathrm{BASF}$ SE, ${ }^{2}$ BASF Coatings GmbH, and ${ }^{3}$ School of Chemistry, University of Bristol.

\section{Notes and references}

1 Y. Wan and D. Zhao, Chem. Rev., 2007, 107, 2821; X. Zhang, et al., Angew. Chem., Int. Ed., 2010, 49, 10101; C. Rodriguez, et al., J. Dispersion Sci. Technol., 2007, 28, 1136.

2 I. Amar-Yuli, et al., Curr. Opin. Colloid Interface Sci., 2009, 14, 21; P. T. Spicer, Curr. Opin. Colloid Interface Sci., 2005, 10, 274; A. Angelova, et al., Acc. Chem. Res., 2011, 44, 147.

3 D. H. Thompson, et al., Proc. SPIE, Organic and Biological Opto-electronics, 1993, vol. 1853, p. 142; S. Laschat, et al., Angew. Chem., Int. Ed., 2007, 46, 4832.

4 T. Ikeda and O. Tsutsumi, Science, 1995, 268, 1873; L. Schmidt-Mende, et al., Science, 2001, 293, 1119; K. Ichimura, Chem. Rev., 2000, 100, 1847.

5 A.-L. Troutier-Thuilliez, et al., Soft Matter, 2011, 7, 4242.

6 R. M. Fuoss and D. Edelson, J. Am. Chem. Soc., 1951, 73, 269.

7 S. Yiv, et al., J. Phys. Chem., 1976, 80, 2651; Y. Okahata and T. J. Kunitake, J. Am. Chem. Soc., 1979, 101, 5231.

8 A. Gulik, et al., J. Mol. Biol., 1985, 182, 131.

9 M. A. Voronin, et al., ACS Appl. Mater. Interfaces, 2011, 3, 402; J. Paczesny, et al., Phys. Chem. Chem. Phys., 2012, 14, 14365; J. K. W. Chui, et al., Beilstein J. Org. Chem., 2011, 7, 1562; N. Nuraje, et al., Prog. Polym. Sci., 2013, 38, 302.

10 K. Liu, et al., Chem.-Eur. J., 2012, 18, 8622; G. Wu, et al., Chem. Sci., 2013, 4, 4486; P. Xing, et al., RSC Adv., 2013, 3, 24776.

11 A. Polidori, et al., ARKIVOC, 2006, iv, 73.

12 J. Guilbot, et al., Langmuir, 2001, 17, 613.

13 J. Wang, et al., J. Sci. Food Agric., 2010, 90, 424; P. Dokic, et al., Prog. Colloid Polym. Sci., 2008, 135, 48.

14 J. C. Roberts, The Chemistry of Paper. Chapter 7, Chemical Additives in the Paper Formation Process, Royal Society of Chemsitry, Herts, UK, 1996, pp. 124-130.

15 D. Tsiourvas, et al., Prog. Colloid Polym. Sci., 1994, 97, 163. 16 J.-H. Fuhrhop, et al., Langmuir, 1985, 1, 387.

17 R. Ramaswamy, et al., J. Appl. Polym. Sci., 1987, 33, 49.

18 C. Y. Zhang, et al., Phys. Rev. E: Stat. Phys., Plasmas, Fluids, Relat. Interdiscip. Top., 1993, 48, 2850; R. Strey and R. Schomäcker, J. Chem. Soc., Faraday Trans., 1990, 86, 2253. 19 N. Satoh and K. Tsujii, J. Phys. Chem., 1987, 91, 6629.

20 J. Cortese, et al., J. Am. Chem. Soc., 2011, 133, 19672.

21 J.-H. Fuhrhop and D. Fritsch, Acc. Chem. Res., 1986, 19, 130. 22 Fig. 15 in: F. B. Rosevear, J. Soc. Cosmet. Chem., 1968, 19, 581. 23 E. O. Kiirend, et al., Crystallogr. Rep., 2002, 47, 849; H. Hoffmann, et al., Langmuir, 1994, 10, 3972; J. Oberdisse, et al., Langmuir, 1996, 12, 1212.

24 S. T. Hyde, in Handbook of Applied Surface and Colloid Chemistry, ed. K. Holmberg, John Wiley \& Sons, 2001, ch. 16, p. 315; A. M. Figueiredo Neto and S. R. A. Salinas, The 
Physics of Lyotropic Liquid Crystals, Oxford University Press, Oxford, 2005, p. 174.

25 G. Porte, et al., J. Phys. II, 1991, 1, 1101.

26 G. Porte, J. Phys.: Condens. Matter, 1992, 4, 8649.
27 M. C. Holmes, in Nanostructured Soft Matter, ed. A. V. Zvelindovsky, Springer, 2007, Part I, ch. 3, p. 99.

28 J. C. Blackburn and P. K. Kilpatrick, Langmuir, 1992, 8, 1679. 29 J. Charvolin and J.-F. Sadoc, J. Phys. Chem., 1988, 92, 5787. 